\title{
Geração da Malha da Traquéia e dos Tubos Bronquiais por Splines Cúbico
}

\section{Cubic Splines for Trachea and Bronchial Tubes Grid Generation}

\author{
Eliandro Rodrigues Cirilo ${ }^{1}$ Álvaro Luis de Bortoli ${ }^{2}$
}

\section{Resumo}

\begin{abstract}
A geração de malhas desempenha um papel importante no desenvolvimento de técnicas numéricas eficientes para a resolução de escoamentos complexos. Dessa forma, o presente trabalho apresenta um método para a geração de malhas em blocos estruturados bidimensionais para geometrias como as de uma traquéia e dos tubos bronquiais. Um conjunto de 55 blocos define a geometria, cujo contorno é definido por Splines Cúbicos. Além disso, essa técnica inova em relação às anteriores, por causa da sua simplicidade e eficiência, em termos da geração de malhas para geometrias muito complexas.
\end{abstract}

Palavras-chave: Splines. Escoamentos. Traquéias e tubos bronquiais

\begin{abstract}
Grid generation plays an important role in the development of efficient numerical techniques for solving complex flows. Therefore, the present work develops a method for bidimensional blocks structured grid generation for geometries such as the trachea and bronchial tubes. A set of 55 blocks completes the geometry, whose contours are defined by cubic splines. Besides, this technique build on early ones because of its simplicity and efficiency in terms of very complex geometry grid generation.
\end{abstract}

Key-words: Splines. Flows. Trachea and bronchial tubes.

\footnotetext{
${ }^{1}$ Professor Assistente B do Dep. de Matemática da UEL. Email: ercirilo@uel.br.

${ }^{2}$ Professor Adjunto IV do Dep. de Matemática Pura e Aplicada da UFRGS. Email: dbortoli@mat.ufrgs.br.
} 


\section{Introdução}

Nos últimos anos, a técnica de simular numericamente as características evolutivas de sistemas complexos vem recebendo muita atenção por parte dos pesquisadores das mais diversas áreas do conhecimento humano. São vários os fatores que permitiram o desenvolvimento da simulação numérica e computacional: a evolução na arquitetura de processamento e armazenamento de dados dos computadores; a disseminação de novos conceitos matemáticos, que, por sua vez, permite a formulação e análise de modelos de fenômenos anteriormente não mensurados; a possibilidade de expressar padrões de comportamento por meio da solução numérica, entre outros. Evidentemente esses não são os únicos fatores, pois seria praticamente impossível descrevêlos na sua totalidade.

A simulação numérica e computacional é fundamental nos mais variados ramos do conhecimento, em particular na Dinâmica dos Fluidos Computacional. A Dinâmica dos Fluidos Computacional trata do estudo de escoamentos de fluidos incompressíveis e compressíveis, com ou sem transferência de calor (PATANKAR, 1981; ANDERSON; TANNEHIL; PLETCHER, 1984), por meio da resolução numérica de modelos matemáticos (DEGREZ, 1996). As equações: de Navier-Stokes, da continuidade, de estado, da temperatura, ou variações destas, podem ser utilizadas na formulação de tais modelos. Por exemplo, se o problema é caracterizado como incompressível e sem transferência de calor, então as equações de NavierStokes serão utilizadas no cálculo da velocidade e a equação de Poisson, que é obtida a partir das equações de Navier-Stokes e da continuidade, será utilizada no cálculo da pressão (WHITFIELD, 1983). Por outro lado se o problema é (por exemplo) caracterizado como compreensível com transferência de calor, então as equações de Navier-Stokes serão utilizadas para o cálculo da velocidade. Além disso, o campo de variações da massa específica será obtido a partir da equação da continuidade, a equação da temperatura para o cálculo das variações de calor. Já para o fechamento do problema a equação de estado será a equação evolutiva da pressão (DE BORTOLI, 2000).

Basicamente, a resolução numérica do modelo matemático se dá em duas fases, primeiramente criase a malha computacional discreta, similar à geometria do problema real (THOMPSON; WARSI; MASTIN, 1985; MALISKA, 1995) e, por conseguinte, são resolvidas numericamente, via métodos numéricos, as equações governantes (DE BORTOLI, 2000; FLETCHER, 1991).

Uma das dificuldades em modelar e analisar o perfil dos escoamentos é a reprodução computacional da geometria, pois a sua complexidade, na maioria das vezes, implica um fator decisório quanto a uma análise mais refinada dos gradientes de interesse.

$\mathrm{O}$ presente trabalho concentrou-se especificamente nos aspectos da criação de uma malha para um problema qualquer, e depois, de forma mais particular, na criação da malha da traquéia e dos tubos bronquiais. Optou-se por esse procedimento porque a representação discreta é complexa e, possivelmente, apresenta gradientes de velocidade, pressão, massa específica e temperatura interessantes de se estudar do ponto de vista numérico. O objetivo em obter a malha da traquéia e dos tubos bronquiais é justificado pelo fato de que muitos problemas patológicos, tais como, por exemplo, a fibrose cística causadora do mal funcionamento de certas glândulas do corpo, provoca a produção de um muco espesso. Esse muco bloqueia os canais bronquiais e ocasiona dificuldade para respirar, tosse crônica e infecções. Por isso, a fibrose cística poderia ser melhor compreendida, se o fluxo nessa geometria fosse descrito em maiores detalhes.

Ao longo do tempo, muitos pesquisadores desenvolveram um rico conjunto de teorias matemáticas capazes de reproduzir computacionalmente, com grande fidelidade, a geometria física dos problemas. Dentre as várias teorias, foram utilizadas, neste trabalho, as equações diferenciais parciais, do tipo elíptica, para discretizar 
o interior da malha computacional. Também se utilizou o método de interpolação polinomial Spline Cúbico (THOMPSON; WARSI; MASTIN, 1985), para representar a fronteira discreta da malha. Esses foram utilizados por causa do seu desempenho computacionale ainda pela rápida similaridade obtida com a geometria física, a partir de poucos pontos conhecidos do domínio.

\section{Equações de Geração das Linhas do Interior da Malha}

Para o presente trabalho optou-se pela discretização estruturada, por possuir vantagens na fácil ordenação dos volumes elementares. Esse método permite a implementação computacional otimizada de muitos métodos de solução, e em coordenadas generalizadas, porque esse sistema permite que a malha computacional seja coincidente com a geometria do problema, sendo o tratamento computacional mais adequado. Certamente, é mais eficiente aplicar metodologias numéricas se a discretização for coincidente na fronteira, neste caso, os volumes elementares são bem definidos nos contornos (MALISKA, 1995), como se vê na Figura 1.

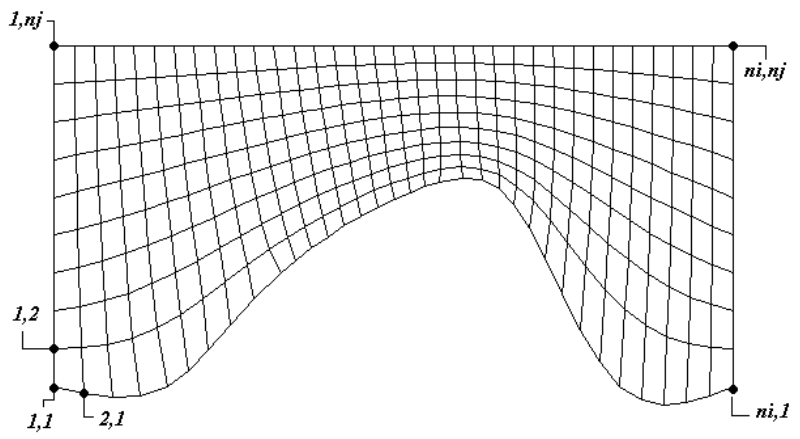

Figura 1 - Malha computacional coincidente com a fronteira.

As equações que permitem gerar as linhas coordenadas nas direções $\xi$ e $\eta$, no interior da malha computacional da Figura 1, como de qualquer outra geometria, são dadas por

$$
\begin{aligned}
\alpha x_{\xi \xi}+\gamma x_{\eta \eta}-2 \beta x_{\xi \eta}+\frac{1}{J^{2}} & \\
\left(P x_{\xi}+Q x_{\eta}\right) & =0
\end{aligned}
$$

$\mathrm{e}$

$$
\begin{aligned}
\alpha y_{\xi \xi}+\gamma y_{\eta \eta}-2 \beta y_{\xi \eta}+\frac{1}{J^{2}} \\
\left(P y_{\xi}+Q y_{\eta}\right)=0
\end{aligned}
$$

onde $x$ e $y$ são as coordenadas cartesianas do domínio físico, $\xi$ e $\eta$ são as coordenadas generalizadas do domínio computacional, $P$ e $Q$ são termos fonte, $\mathrm{e}$

$$
\begin{gathered}
J=x_{\xi} y_{\eta}-x_{\eta} y_{\xi} \\
\alpha=x_{\eta}^{2}+y_{\eta}^{2} \\
\beta=x_{\eta} x_{\xi}+y_{\eta} y_{\xi} \\
\gamma=x_{\xi}^{2}+y_{\xi}^{2}
\end{gathered}
$$

onde $x_{\xi}, x_{\eta}, y_{\xi}$ e $y_{\eta}$ denotam derivas parciais.

As equações (1) e (2) são EDP's elípticas que consistem em um sistema de equações de geração de malhas para as coordenadas físicas no plano transformado. O leitor interessado nos pormenores da obtenção destas equações pode consultar (DE BORTOLI, 2000; MALISKA, 1995).

Portanto, a solução das equações (1) e (2) sujeitas a condições iniciais e de contorno, sendo esta última condição pormenorizada a seguir, fornecerão as linhas $\xi$ e $\eta$, que superpostas, nos dá a malha computacional.

Os termos fonte $P$ e $Q$ são responsáveis pela atração entre linhas coordenadas, cujas expressões são,

$$
\begin{array}{r}
P(\xi, \eta)=-\sum_{j=1}^{n j} a_{j} \operatorname{sign}\left(\xi-\xi_{j}\right) \cdot e^{-c_{j}\left|\xi-\xi_{j}\right|} \\
-\sum_{i=1}^{n i} b_{i} \operatorname{sign}\left(\xi-\xi_{i}\right) \cdot e^{-d_{i} \sqrt{\left(\xi-\xi_{i}\right)^{2}+\left(n-n_{i}\right)^{2}}}
\end{array}
$$

$\mathrm{e}$

$$
\begin{aligned}
& Q(\xi, \eta)=-\sum_{j=1}^{n j} a_{j} \operatorname{sign}\left(\eta-\eta_{j}\right) \cdot e^{-c_{j}\left|\eta-\eta_{j}\right|} \\
& -\sum_{i=1}^{n i} b_{i} \operatorname{sign}\left(\eta-\eta_{i}\right) \cdot e^{-d_{i} \sqrt{\left(\xi-\xi_{i}\right)^{2}+\left(\eta-\eta_{i}\right)^{2}}}
\end{aligned}
$$


Os índices dos somatórios $n i$ e $n j$ representam o número total de linhas nas direções $\xi$ e $\eta$ respectivamente; e $a_{j}, b_{i}, c_{j}, d_{i}$ são números reais ajustados via experimentação numérica, procurando atrair as linhas $\xi$ e $\eta$ para as linhas $\xi_{i}$ e $\eta_{i}$. Para outros pormenores, o leitor interessado pode consultar (MALISKA, 1995; DE BORTOLI, 2000; THOMPSON; WARSI; MASTIN, 1985). O ajuste desses coeficientes depende das características de cada problema a ser resolvido. Por exemplo, podese visualizar na Figura 2 a atração das linhas para o contorno inferior para todas as linhas, onde os gradientes de velocidade, massa específica, pressão e temperatura são melhores de ser captados na malha da Figura 2-b do que na malha da Figura 2-a.

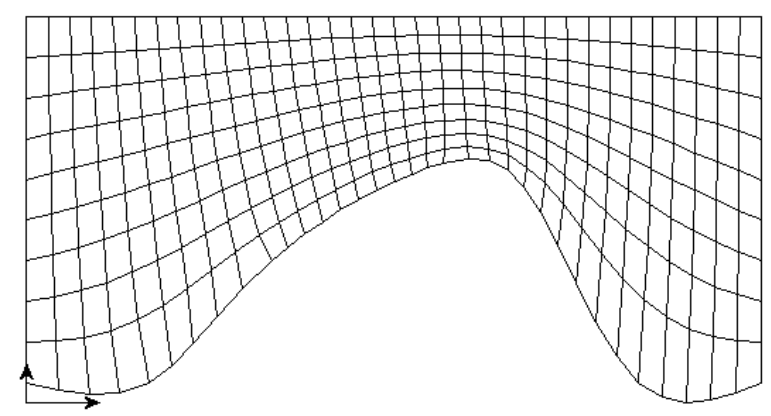

(a)

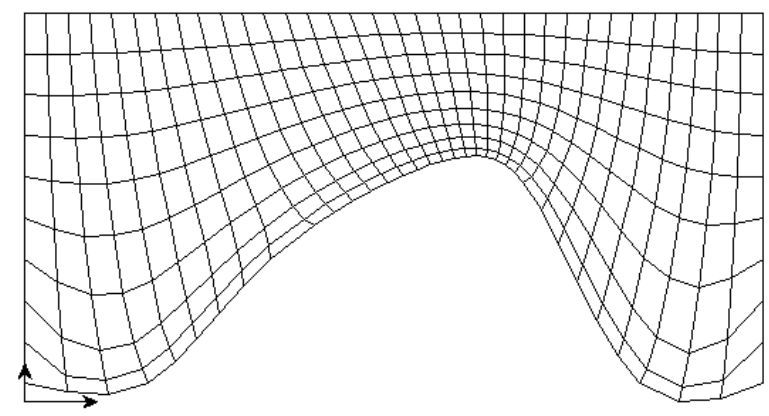

(b)

Figura 2 - (a) Malha sem concentração de linhas, (b) Malha com concentração de linhas.

Observe que as equações (1) e (2) podem ser escritas segundo uma variável $\phi$ genérica como (DE BORTOLI, 2000; MALISKA, 1995),

$$
\begin{aligned}
& \alpha \phi_{\xi \xi}+\gamma \phi_{\eta \eta}-2 \beta \phi_{\xi \eta}+\frac{1}{J^{2}} \\
&\left(P \phi_{\xi}+Q \phi_{\eta}\right)=0
\end{aligned}
$$

Assim o método de diferenças finitas, aproxima as derivadas parciais presentes na equação (9) por diferenças centrais, e dele resulta o sistema linear dado na forma

$$
\begin{aligned}
& \phi_{i, j}=\frac{1}{a_{i, j}} \cdot\left(a_{i+1, j} \cdot \phi_{i+1, j}+a_{i-1, j} \cdot \phi_{i-1, j}\right. \\
& +a_{i, j+1} \cdot \phi_{i, j+1}+a_{i, j-1} \cdot \phi_{i, j-1}+a_{i+1, j+1} \cdot \phi_{i+1, j+1} \\
& +a_{i-1, j+1} \cdot \phi_{i-1, j+1}+a_{i+1, j-1} \cdot \phi_{i+1, j-1}+ \\
& \left.a_{i-1, j-1} \cdot \phi_{i-1, j-1}\right)
\end{aligned}
$$

onde

$$
\begin{array}{ll}
a_{i, j}=2 \cdot \alpha+2 \cdot \gamma & a_{i+1, j}=\alpha+\frac{P}{2 \cdot J^{2}} \\
a_{i-1, j}=\alpha-\frac{P}{2 \cdot J^{2}} & a_{i, j+1}=\gamma+\frac{Q}{2 \cdot J^{2}} \\
a_{i-1, j-1}=a_{i+1, j+1} & a_{i+1, j+1}=-\frac{\beta}{2} \\
a_{i+1, j-1}=a_{i-1, j+1} & a_{i, j-1}=\gamma-\frac{Q}{2 \cdot J^{2}} \\
a_{i-1, j+1}=-a_{i+1, j+1} &
\end{array}
$$

O sistema linear (10) vai ser resolvido pelo método de Gauss-Seidel com relaxação (SMITH, 1990), cujo coeficiente de relaxação mais apropriado foi da ordem de 0,8 .

\section{Equações de Geração do Contorno da Malha Computacional Via Spline Cúbico}

O aumento da capacidade de processamento e armazenamento dos computadores atuais viabilizou o uso da interpolação em problemas complexos, que é fundamental para a geração de malhas no sistema de coordenadas generalizadas. Dentre as várias técnicas, a interpolação polinomial é a mais utilizada, por possuir vantagens na simplicidade computacional de seus cálculos.

O método de interpolação polinomial de Lagrange é vantajoso do ponto de vista da simplicidade 
matemática e da implementação computacional, porém, oscilações surgem com o aumento do número de pontos a serem interpolados (SPERANDIO; MENDES; MONKEN, 2003), de modo que o contorno a ser modelado pode não ser representativo em relação ao contorno real.

A interpolação polinomial Spline Cúbica Parametrizada surge como uma alternativa viável, pois as imposições feitas sobre o polinômio interpolador minimizam as oscilações (RUGGIERO; LOPES, 1996). Adicionalmente, essa técnica é simples do ponto de vista matemático e sua implementação computacional não é complicada. Dessa forma, o contorno da geometria a ser modelado é bem representado, portanto esta foi a metodologia empregada neste trabalho.

Considere que as coordenadas de um dado contorno sejam conhecidas pelos pares ordenados $\left(x_{n}, y_{n}\right)$ tabelados em $(n+1)$ pontos.

Tabela 1. Coordenadas de um contorno qualquer parametrizado em $t$.

\begin{tabular}{l|llllll}
\hline$t$ & 1 & 2 & 3 & & $n$ & $(n+1)$ \\
\hline$x$ & $x_{1}$ & $x_{2}$ & $x_{3}$ & $\ldots .$. & $x_{n}$ & $x_{(n+1)}$ \\
$y$ & $y_{1}$ & $y_{2}$ & $y_{3}$ & $\ldots .$. & $y_{n}$ & $y_{(n+1)}$ \\
\hline
\end{tabular}

O referido contorno pode ser ajustado via polinômios cúbicos parametrizados em $t$, conforme as expressões:

$$
X(h)=a_{3_{t}}(h-t)^{3}+a_{2_{t}}(h-t)^{2}+a_{1_{t}}(h-t)+a_{0_{t}}
$$

$\mathrm{e}$

$$
Y(h)=b_{3_{t}}(h-t)^{3}+b_{2_{t}}(h-t)^{2}+b_{1_{t}}(h-t)+b_{0_{t}}
$$

sendo $h$ um número real.

A variável $h$ é ajustada na medida que se deseja efetuar, ou não, a concentração de pontos à esquerda ou à direita de um ponto base. Por exemplo, a Figura 3 ilustra a obtenção do contorno inferior da malha dessa forma. No intervalo de extremos $t=1$ e $t=2$, não foi aplicada a concentração de pontos, ou seja, a marcação dos pontos 2 e 3 , foram feitas tomando $h=\frac{4}{3}$ e $h=\frac{5}{3}$ respectivamente, tornando os pontos do intervalo igualmente espaçados. De modo análogo, fez-se a marcação dos pontos nos intervalos [2,3], $[5,6]$ e $[6,7]$. Por outro lado, para o intervalo $[3,4]$, foi aplicada a concentração de pontos na direção do ponto 20 , ou seja, a marcação dos pontos 10 a 19 foram feitas com $h$ decrescendo exponencialmente. De modo análogo, fez-se concentração de pontos, do intervalo [4,5], na direção do ponto 20 .

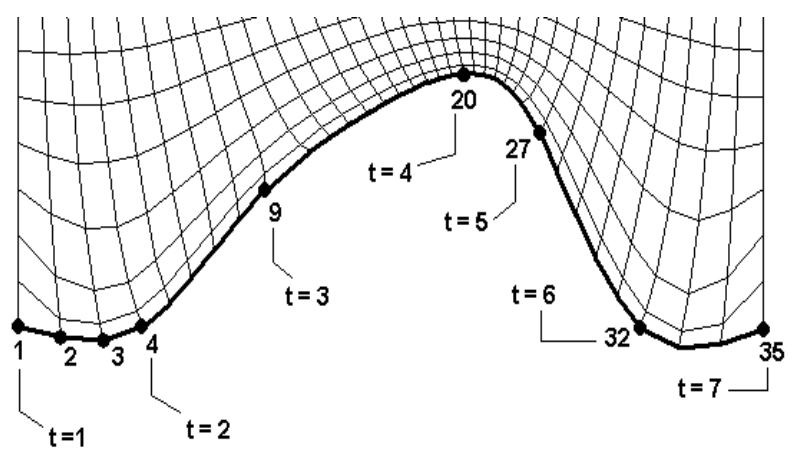

Figura 3 - Contorno inferior obtido por Spline Cúbico Parametrizado.

Conforme Ruggiero e Lopes (1996), considerando o método spline cúbico natural, os coeficientes dos polinômios (12) e (13) são determinados a partir da resolução dos respectivos sistemas lineares:

$$
\begin{aligned}
& g_{k-1}^{X}+4 g_{k}^{X}+g_{k+1}^{X}=m_{k}^{X} \\
& g_{k-1}^{Y}+4 g_{k}^{Y}+g_{k+1}^{Y}=m_{k}^{Y},
\end{aligned}
$$

para $k=2, \ldots, n$, onde

$$
\begin{aligned}
& m_{k}^{X}=6\left(x_{k+1}-2 x_{k}+x_{k-1}\right) \\
& m_{k}^{Y}=6\left(y_{k+1}-2 y_{k}+y_{k-1}\right)
\end{aligned}
$$

Pode-se mostrar então que os coeficientes de (12) e (13) são 


$$
\begin{aligned}
& a_{3_{k}}=\frac{1}{6}\left(g_{k}^{X}-g_{k-1}^{X}\right) \\
& b_{3_{k}}=\frac{1}{6}\left(g_{k}^{Y}-g_{k-1}^{Y}\right) \\
& a_{2_{k}}=\frac{1}{2} g_{k}^{X} \\
& b_{2_{k}}=\frac{1}{2} g_{k}^{Y} \\
& a_{1_{k}}=x_{k}-x_{k-1}+\frac{1}{3} g_{k}^{X}+\frac{1}{6} g_{k-1}^{X} \\
& b_{1_{k}}=y_{k}-y_{k-1}+\frac{1}{3} g_{k}^{Y}+\frac{1}{6} g_{k-1}^{Y} \\
& a_{0_{k}}=x_{k} \\
& b_{0_{k}}=y_{k}
\end{aligned}
$$

Os sistemas lineares (14) e (15) são tridiagonais e, deste fato, aplicou-se o método de Thomas ou TDMA (TriDiagonal Matrix Algorithm) (MALISKA, 1995) na resolução dos mesmos, pois este método tira proveito da estrutura tridiagonal da matriz dos coeficientes dos sistemas, simplificando significativamente os cálculos. Basicamente, o método, num primeiro momento, faz a eliminação dos termos abaixo da diagonal principal e após, num segundo momento, faz substituições ordenadas calculando os valores dos termos presentes no vetor incógnita. O método reduz consideravelmente o uso de memória (DE BORTOLI, 2000).

\section{Resultados}

A equação (10) é resolvida numericamente pelo método de Gauss-Seidel com relaxação para $\phi=x$ e $\phi=y$, a partir do conhecimento de todo o contorno do domínio. O contorno, obtido a partir de (12) e (13), modela computacionalmente a geometria do problema de maneira discreta através da malha.

Um fator relevante para muitos problemas reais é o seguinte: gerar a malha em um único bloco, mesmo com a metodologia descrita neste trabalho, não permite captar a complexidade da geometria, e por sua vez, o resultado numérico dos cálculos do escoamento poderão não estar modelando adequadamente o escoamento real. Logo surge a necessidade de utilizar a técnica multiblocos (DE BORTOLI, 2000). O código gerador de malhas desenvolvido permite gerar malhas tipo multiblocos. Primeiramente, se faz uma análise qualitativa de quais e quantas sub-malhas (blocos) a malha global seria composta. Após isso, para o primeiro bloco, definese o contorno via Spline Cúbico Parametrizado, e obtém-se o interior resolvendo as equações (1) e (2), tendo ao final, um arquivo de dados de localização espacial de todos os pontos do bloco. Repete-se o mesmo procedimento com todos os blocos, e ao final são lidos os blocos e gravados em um arquivo de dados que é a malha global. A propósito, o emparelhamento de blocos faz-se a partir da leitura dos pontos extremos comuns entre blocos, que são admitidos no novo bloco a ser obtido, Figura 4.

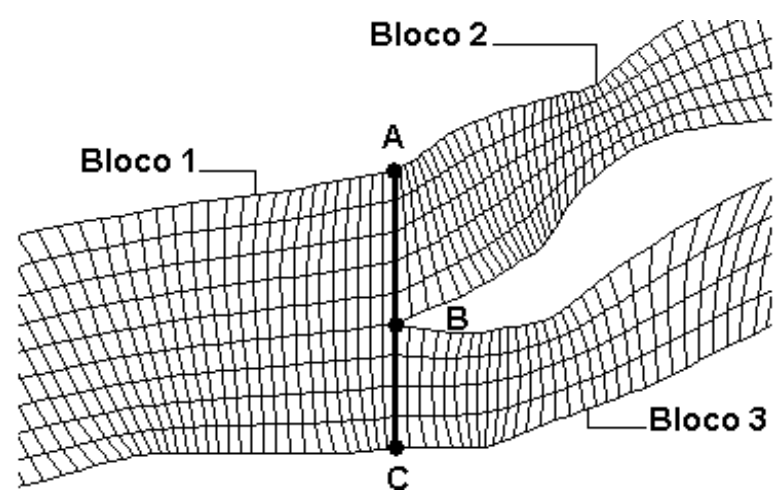

Figura 4 - Junção de três blocos.

No caso da figura 4, as coordenadas de localização espacial do ponto A de rótulo $\left(n_{i}, n_{j}\right)$ para o bloco 1 , serão rotuladas como $\left(1, n_{j}\right)$ no bloco 2. As 
coordenadas do ponto $\mathrm{B}$, de rótulo $\left(n_{i}, \frac{n_{j}}{2}\right)$ para o bloco 1 , serão $(1,1)$ no bloco 2 e $\left(1, n_{j}\right)$ no bloco 3 . Finalmente o ponto $\mathrm{C}$ de rótulo $\left(n_{i}, 1\right)$ para o bloco 1 , será $(1,1)$ para o bloco 3 .

Vale ressaltar que o código permite gerar blocos de malha grossa e outros de malha fina. Se ocorrer a junção entre blocos com essa natureza, então haverá a necessidade de se fazer o uso de funções de interpolação se estiverem sendo calculadas variáveis do tipo velocidade, pressão e etc. O gerador de malhas também está habilitado para se permitir calcular escoamentos por meio da técnica multigrid.

Como estudo de caso, gerou-se, no presente trabalho, a malha bidimensional de uma parte do sistema respiratório constituído pela traquéia, brônquios e tubos bronquiais. A traquéia é um tubo cartilaginoso e membranoso que se estende da laringe, ao nível da sexta vértebra cervical, até a borda cranial da quinta vértebra torácica, onde se divide nos dois brônquios. O brônquio direito é mais largo, mais curto e tem menor angulação em sua divergência da traquéia que o esquerdo. Já o brônquio esquerdo tem menor calibre, mas possui aproximadamente o dobro do comprimento do direito (GRAY, 1988), Figura 5.

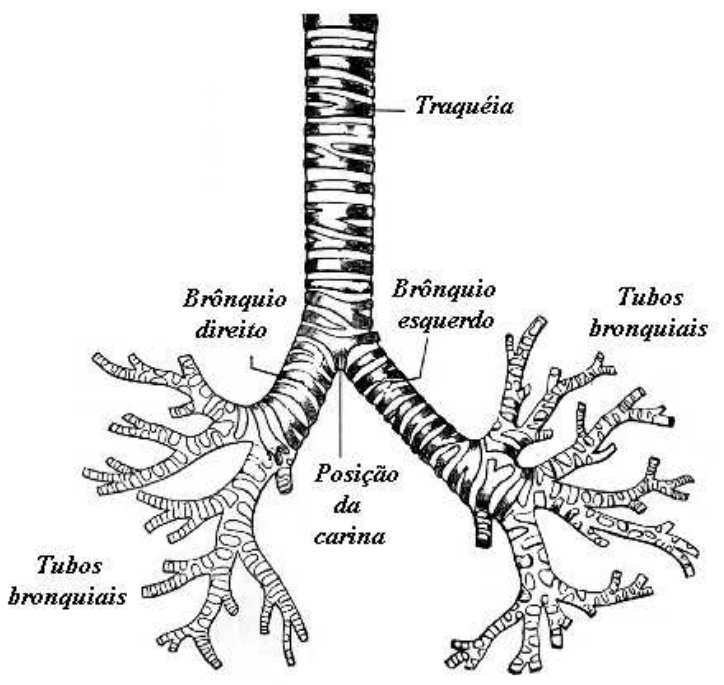

Figura 5 - Geometria da Traquéia e dos Brônquios (GRAY, 1988).

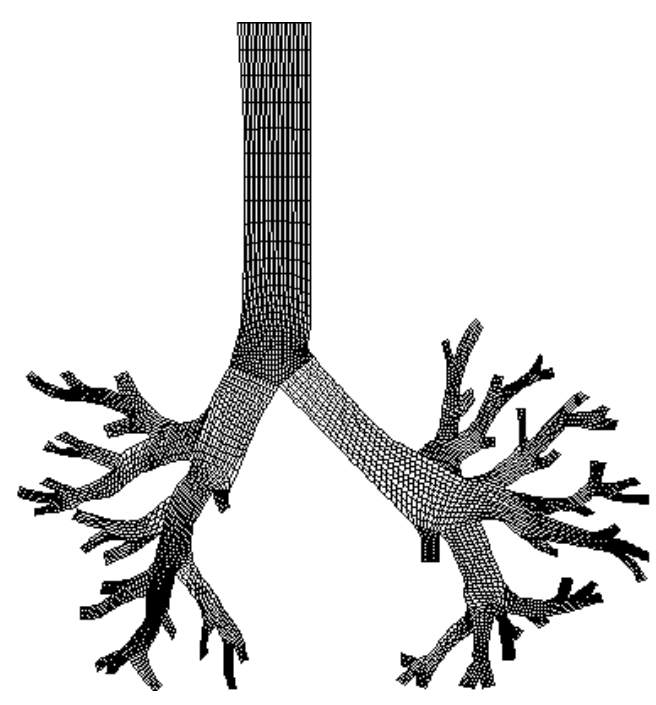

Figura 6 - Malha da Traquéia e dos Brônquios.

A malha computacional da traquéia e brônquios, que modela a geometria da Figura 5, foi gerada tipo multiblocos, num total de 55 blocos e 7082 elementos, em 6 segundos de processamento num computador k7-750MHz (Figura 6), e cada ramificação corresponde a um bloco.

Observe que na junção da traquéia com os brônquios direito e esquerdo Figura 7, há a necessidade de concentração de linhas na malha, porque ocorrerá desvio abrupto de fluxo do fluido que entra para os brônquios, logo os gradientes de pressão e velocidade serão expressivos nesta região. Para este caso, a concentração na malha, foi obtida fazendo $Q(\xi, \eta)=0$, adequando os coeficientes de $P(\xi, \eta)$, aumentando a quantidade de pontos nesta região e ajustando a variável $h$ das equações (12) e (13).

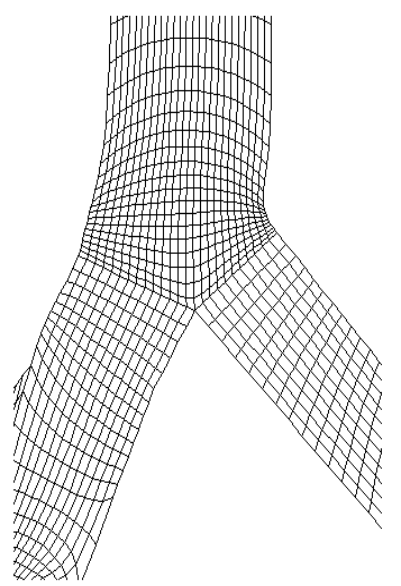

Figura 7-Concentração de linhas na saída da traquéia para os brônquios. 
De modo análogo, na criação computacional dos brônquios esquerdo e direito, e conseqüentemente os tubos bronquiais, figuras 8 e 9 , necessitou-se também realizar a concentração de linhas próximas às suas entradas.

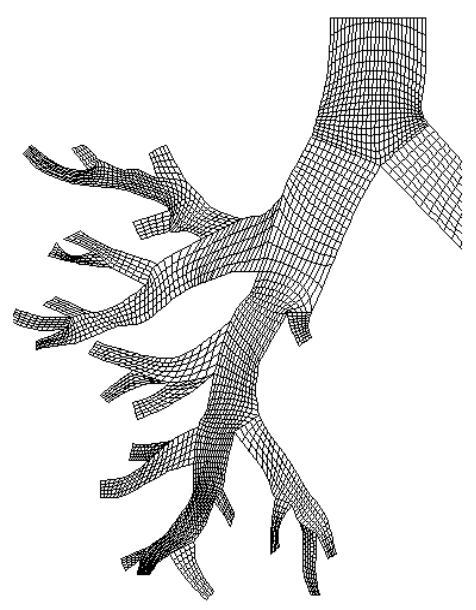

Figura 8 - Brônquio direito e tubos bronquiais.

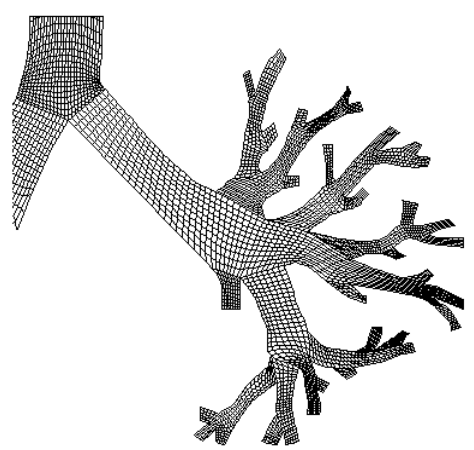

Figura 9 - Brônquio esquerdo e tubos bronquiais.

Assim como na saída da traquéia, ocorrerão gradientes de pressão e velocidade naquelas regiões e a captação destas flutuações, numericamente, permitirão uma análise mais refinada do perfil do escoamento do fluido, e conseqüentemente um diagnóstico mais completo, do ponto de vista patológico, poderá ser realizado.

\section{Considerações Finais}

A geração de malhas de geometrias complexas constitui-se numa delicada e importante etapa do cálculo do escoamento de fluidos, pois se a malha gerada for não representativa, imprecisões quanto aos resultados do escoamento poderão ser não- representativos (DE BORTOLI, 2000; MALISKA, 1995).

Com o desenvolvimento deste trabalho, a técnica, como foi exposta, se mostra simples matematicamente e computacionalmente. $\mathrm{O}$ contorno pode ser modelado por Spline Parametrizado, a partir de uma quantidade pequena de pontos conhecidos. A localização dos pontos interiores é obtida rapidamente a partir da resolução das equações elípticas de geração de malhas. Além disso, a possibilidade de gerar malhas multiblocos permite tratar problemas razoavelmente complexos.

O próximo passo será tratar um modelo matemático que descreva o escoamento dentro da traquéia e dos tubos bronquiais em regime laminar, e resolvê-lo numericamente pelo método dos volumes finitos, e comparar os resultados encontrados com os da literatura existentes.

\section{Referências}

ANDERSON, D. A.; TANNEHIL, J. C.; PLETCHER, R. H. Computational fluid mechanics and heat transfer. New York: McGraw-Hill, 1984.

DE BORTOLI, A. L. Introdução à dinâmica de fluidos computacional. Porto Alegre: UFRGS, 2000.

DEGREZ, G. Implicit time-dependent methods for inviscid and viscous compressible flows. RhodeSt-Genese, Belgium: Von Karman Institute for Fluid Dynamics, 1996.

FLETCHER, C. A. J. Computational techniques for fluid dynamics. Berlin: Springer-Verlag, 1991.

GRAY, F. R. S. H. Anatomia. Rio de Janeiro: Guanabara Koogan, 1988.

MALISKA, C. R. Transferência de calor e mecânica dos fluidos computacional, fundamentos e coordenadas generalizadas. Rio de Janeiro: LTC, 1995. 
PATANKAR, S. V. Numerical heat transfer and fluid flow. New York: McGraw-Hill, 1981.

RUGGIERO, M. A. G.; LOPES, V. L. R. Cálculo numérico: aspectos teóricos e computacionais. São Paulo: Makron, 1996.

SMITH, G. D. Numerical solution of partial differential equations: finite difference methods. New York: Oxford University Press, 1990.

SPERANDIO, D.; MENDES, J. T.; MONKEN, L. H. Cálculo numérico: características matemáticas e computacionais dos métodos numéricos. São Paulo: Prentice Hall, 2003.

THOMPSON, J. F.; WARSI, Z. U. A.; MASTIN, C. W. Numerical grid generation: foundations and applications. New York: Elsevier Science Publishing, 1985.

WHITFIELD, D. L. Three-dimensional unsteady euler equations solutions using flux vector splitting. Mississippi: Mississippi State University, 1983. 